\title{
Emisiones de dióxido de carbono en estratos de mangle en la Amazonia Oriental Brasileña
}

\author{
Nelson Castellón Rodríguez ${ }^{1}$, Maria Isabel Vitorino², José F. Berrêdo ${ }^{3}$, \\ Mário Augusto Gonçalves Jardim ${ }^{4} \&$ Artur Gustavo Oliveira de Miranda ${ }^{5}$ \\ 1. Programa de Pós-Graduação em Ciências Ambientais, Universidade Federal do Pará, Brasil; castellonnr@gmail.com \\ 2. Departamento de Meteorologia, Universidade Federal do Pará, Brasil; vitorino@ufpa.br \\ 3. Coordenação de Ciências da Terra e Ecologia, Museu Paraense Emilio Goeldi, Pará; berredo@museu-goeldi.br \\ 4. Museu Paraense Emilio Goeldi, Coordenação de Botânica, Pará; jardim@museu-goeldi.br \\ 5. Laboratório de Análises de Imagens do Trópico Úmido, Universidade Federal do Pará, Brasil; \\ arturgustavomiranda@gmail.com
}

Recibido 12-II-2019. Corregido 20-VIII-2019. Aceptado 29-II-2020.

\begin{abstract}
Carbon dioxide emissions in mangrove strata of the Eastern Brazilian Amazonia. Introduction: Carbon dioxide emissions from mangrove soils have a potential impact on the global carbon balance. They are related to anthropic actions and natural processes with interspecific interactions involving physical-chemical and environmental variables. Objective: In this research, the seasonality of carbon dioxide emissions related to different strata of mangrove vegetation, soil physical-chemical makeup and physical environmental factors were evaluated. Methods: Nine plots of $20 \times 20 \mathrm{~m}$ were demarcated (three for each of the three vegetation strata) in the Experimental Site of the Federal Rural University of the Amazon and the Federal University of Pará in Cuiarana, Salinópolis, Pará, Brazil. Duplicate soil samples were taken from each plot during three consecutive seasonal periods and analyzed in the laboratory. Carbon dioxide emissions were monitored for 88 days through the basal breathing of the soil methodology; other variables evaluated were soil moisture and temperature, hydrogenic potential, redox potential, carbon and nitrogen of the microbial biomass, organic matter and composition of sand, silt and clay. Precipitation data was obtained from the CMORPH technique of the Climate Forecast Center - NOAA. Information on tides was obtained from the Brazilian Navy's Fundeadouro de Salinópolis. Results: The results showed that the highest carbon dioxide emissions occurred in the rainy season 2017 on average $7.5\left(14.5 \mathrm{TCO}_{2}\right.$ ha/year) $\mathrm{mg} / 100 \mathrm{~cm}^{3}$. With $10.5 \mathrm{mg} / 100 \mathrm{~cm}^{3}\left(21 \mathrm{TCO}_{2}\right.$ ha/year), the adult stratum was the largest source of emissions. The highest seasonal correlations of the emissions in relation to the incubation intervals occurred in the rainy season, in the adult stratum the days 1,2,3,4,3,3 and 5. Using principal component analysis (PCA) it was found that the highest correlations of carbon dioxide emissions and physical-chemical variables occurred in the adult stratum with $56 \%$ variance. The highest correlations were found with the variables soil moisture, $\mathrm{Ph}$, organic matter, carbon and microbial nitrogen. The Kruskal-Wallis test corroborated these results, indicating significant differences between vegetation strata and $\mathrm{CO}_{2}$ emissions $(\mathrm{P}=0.0170)$; and the Tukey test confirmed greater statistical importance of the adult mangrove in relation to the other strata $(\mathrm{P}=0.0140)$. Conclusions: In the three analyzed stations, the highest emissions occurred in the rainy period with an average of $14.5 \mathrm{TCO}_{2}$ ha/year and the adult stratum was responsible for the highest emissions registering $21 \mathrm{TCO}_{2}$ ha/year difference that was statistically significant with the other strata $(\mathrm{P}=0.0140)$.
\end{abstract}

Key words: carbon flow; climate change; ecosystem; microorganisms; sediments.

Castellón Rodríguez, N.. Vitorino, M.I., Berrêdo, J.F., Gonçalves Jardim M.A., \& Oliveira de Miranda, A.G. (2020). Emisiones de dióxido de carbono en estratos de mangle en la Amazonia Oriental Brasileña. Revista de Biología Tropical, 68(1), 289-299. 
Aproximadamente el $30 \%$ de dióxido de carbono $\left(\mathrm{CO}_{2}\right)$ liberado a la atmósfera, proviene de la destrucción de los ecosistemas naturales contribuyendo con el calentamiento global (Houghton, 2003). Las altas tasas de deforestación se destacan como la principal causa que está provocando la pérdida de biomasa aérea y la liberación del $\mathrm{CO}_{2}$ a través de la respiración basal del suelo (RBS) como lo demuestran De Souza Costa, Bayer, Zanatta, y Mielniczuk (2008) y Troxler et al. (2015). Estudios de Stockmann et al. (2013) indican que la acumulación de carbono en el suelo es producto de la entrada de los compuestos orgánicos mediante el secuestro del $\mathrm{CO}_{2}$ atmosférico y las emisiones son causadas por la erosión, lixiviación y oxidación resultante de la actividad metabólica de los macro y micro organismos (Doran \& Parkin, 1994).

Las emisiones de $\mathrm{CO}_{2}$ producto de los procesos naturales, ocurren por la respiración microbiana y radicular, la deposición y descomposición del material orgánico, favorecido por las propiedades físicas y químicas del suelo, factores ambientales y biológicos, sus tasas de descomposición y la vegetación (Reef, Feller, \& Lovelock, 2010; Bond-Lamberty \& Thomson, 2010; Kim, Vargas, Bond-Lamberty, \& Turetsky, 2012; Fiedler, Buczko, Jurasinski, \& Glatzel, 2015). Ecosistemas como los manglares que se caracterizan por un intenso reciclaje de materia orgánica en sus sedimentos, representan un impacto potencial para el equilibrio global del carbono tanto por la acción antrópica o natural, la pérdida anual se estima en 340000 y 980000 ha de mangle (Holguin, Vazquez, \& Bashan, 2001; Sifleet, Pendleton, \& Murray, 2011; Alongi, 2012).

Las emisiones globales por la degradación de los manglares representan hasta 0.12 pentagramas de carbono al año, el $10 \%$ de las emisiones asociadas a los bosques tropicales, aunque solo ocupan el $1 \%$ del área terrestre (Donato et al., 2011). En la literatura se reportan una gama de estudios de las emisiones de $\mathrm{CO}_{2}$, sin embargo, investigaciones por estratos de vegetación son limitadas a pesar de ser un ecosistema que es influenciado en su vegetación y sus características físico-químicas por la marea (Souza, Guedes, Oliveira, \& Santos, 1996).

La hipótesis probada es que las emisiones de $\mathrm{CO}_{2}$ en los suelos de los manglares difieren entre los estratos de vegetación y son influenciadas por la estacionalidad, las características físico-químicas y los factores ambientales. Esta investigación tuvo como objetivo evaluar la estacionalidad y espacialidad de las emisiones de $\mathrm{CO}_{2}$ y su relación con las variables físicoquímicas del suelo y ambientales en un manglar de la Amazonia Oriental brasileña.

\section{MATERIAL Y MÉTODOS}

Área de estudio: El estudio se localizó en el Municipio de Salinópolis, en la comunidad de Cuiarana, en la región nordeste del estado de Pará, Brasil (47²8’47” E \& 066’80” S), en la Microrregión Salgado según la clasificación fisiográfica de (Schaeffer-Novelli et al., 1990).

Análisis de variables ambientales: Los datos de precipitación fueron obtenidos de la técnica CMORPH del Centro de Previsión del Clima de la NOAA (https:// cpc.ncep.noaa. gov/products/.../cmorph) en la escala de media hora y resolución espacial de $8 \mathrm{~km}$ para enero de 1998 a diciembre de 2017 (Joyce, Janowiak, Arkin, \& Xie, 2004). Se calcularon las medias totales mensuales y posteriormente los promedios climatológicos mensuales utilizados en las anomalías de la precipitación de enero a diciembre 2016 y 2017 y se calcularon sustrayendo del acumulado mensual de cada año la media climatológica mensual del período 1998/2017 a partir de la siguiente ecuación.

$$
\overline{\mathrm{A}} J=\sum_{1998}^{2017} \frac{J i}{N^{\circ} \text { años }}(1)
$$

Donde: $\bar{A} \mathrm{j}=$ Anomalía de la precipitación en enero. El cálculo de la altura media mensual de la marea se efectuó a partir de la información del Fundeadouro de Salinópolis (Marina brasilera) para cuatro medidas diarias para el período 2016/2017, (https://surfguru.com.br/ previsión/mare/20520). Se tomaron los valores 
correspondientes a la marea de sizigia (luna llena y nueva).

Diseño del muestreo y medición de las emisiones de $\mathrm{CO}_{2}$ : a partir de tres parcelas 20x20 m demarcadas por estrato, mangle joven (1-3 $\mathrm{m}$ de altura), intermediario (3.1-6 $\mathrm{m}$ de altura) y adulto ( $\geq 6 \mathrm{~m}$ de altura), fueron recolectadas dos muestras de suelo de $400 \mathrm{~g}$ en cada parcela a una profundidad de $0-20 \mathrm{~cm}$. Esto durante el período lluvioso de 2017 (mayo) y menos lluvioso de 2016/2017 (noviembre). En total se analizaron 36 muestras en el Laboratorio de Suelo de la Coordinación de Ciencias de la Tierra y Ecología del Museo Paraense Emilio Goeldi.

Las emisiones de $\mathrm{CO}_{2}$ se midieron a través de la metodología respiración basal del suelo (RBS), mediante la incubación en el laboratorio de $50 \mathrm{~g}$ de suelo y $30 \mathrm{ml}$ de solución de $\mathrm{NaOH} 0.5 \mathrm{~mol}^{-1}$ en recipientes plásticos herméticos a $25^{\circ} \mathrm{C}$ (Matos, 2005). $\mathrm{El} \mathrm{CO}_{2}$ fue medido por un período de 88 días, con titulaciones los días 1,2,3,4 y posteriormente a intervalos de $3,3,5,6,7,10,14,16$ y 22 días luego de la incubación, proceso replicado durante tres periodos estacionales. En la titulación se utilizaron $10 \mathrm{ml}$ de $\mathrm{NaOH}$ y $10 \mathrm{ml}$ de $\mathrm{BaCl}_{2} 0.05 \mathrm{~mol} \mathrm{~L}^{-1}$ para la precipitación de los carbonatos y añadida la solución indicadora fenolftaleína al $1 \%$. Se tituló con ácido clorhídrico (HCL) de forma duplicada. El $\mathrm{CO}_{2}$ liberado fue expresado en $\mathrm{mg} / 100 \mathrm{~cm}^{3}$ y cuantificado según Anderson y Domsch (1993).

$$
\mathrm{C}-\mathrm{CO}_{2}=(\mathrm{B}-\mathrm{V}) \times \mathrm{M} \times 12 \times\left(\frac{\mathrm{v} 1}{\mathrm{v} 2}\right)
$$

Donde; $\mathrm{B}=$ Volumen del $\mathrm{HCl}$ en blanco $(\mathrm{mL})$; $\mathrm{V}=$ Volumen de $\mathrm{HCl}$ usado en la muestra $(\mathrm{mL})$; $\mathrm{M}=$ Concentración real del $\mathrm{HCl}\left(\mathrm{mol} \mathrm{L}^{-1}\right) ; 12$ $=$ Peso equivalente del carbono; $\mathrm{v} 1=$ Volumen total de $\mathrm{NaOH}$ utilizado en la captura de $\mathrm{CO}_{2}$ $(\mathrm{mL})$; v2 = Volumen de $\mathrm{NaOH}$ utilizado en la titulación $(\mathrm{mL})$. Los resultados se expresaron en toneladas de $\mathrm{CO}_{2}$ por hectárea por año $\left(\mathrm{TCO}_{2}\right.$ ha/año). El cálculo comprende, conversiones de miligramos en cien centímetros cúbicos $\left(\mathrm{mg} / 100 \mathrm{~cm}^{3}\right)$ para miligramos por decímetro cúbico $\left(\mathrm{mg} / \mathrm{dm}^{3}\right)$ dividido entre el factor 500 para $\mathrm{TCO}_{2}$ ha/año.

Variables físico-químicas: Los valores de temperatura del suelo corresponden a los días de medición de las emisiones de $\mathrm{CO}_{2}$ y obtenidos con un termómetro portátil TASI 8601 , a nivel superficial. La humedad gravimétrica en el suelo fue determinada en el laboratorio a través de la relación del peso húmedo y peso seco de la muestra. En campo, se midieron los valores del Eh $(\mathrm{mV})$ y $\mathrm{pH}$ con un equipo Metrhom 744 , en el Eh se utilizó un electrodo de platino con valor de lectura corregido con la adición del valor del electrodo de referencia de calomelano (+244 mV). Lecturas de $\mathrm{pH}$ se hicieron con un electrodo de vidrio calibrado con soluciones patrones de $\mathrm{pH}=4.0$ y 7.0, insertado directamente en el sedimento (Berrêdo, Costa, Vilhena, \& Santos, 2018). La salinidad intersticial fue determinada con un refractómetro de campo, extrayendo bajo presión un pequeño volumen de agua. La granulometría se determinó por el método de la división de la masa de muestra seca $(\mathrm{g}) /$ volúmenes de muestra $\left(\mathrm{m}^{3}\right)$. El carbono y el nitrógeno de la biomasa microbiana se analizaron mediante el método irradiado y no irradiado. El Carbono en la Biomasa Microbiana (CBM) se calculó de acuerdo a Matos (2005).

$$
\text { Cmicrobiana }(\mu \mathrm{g} \cdot \mathrm{g}-1)=\frac{(\mathrm{CI}-\mathrm{C} \tilde{\mathrm{N} I})}{\mathrm{Kc}}
$$

Donde; $\mathrm{CI}=$ muestra irradiada; $\mathrm{CN} \mathrm{I}=$ muestra no irradiada; $\mathrm{Kc}=0.33$ para el método de irradiación extracción de CBM.

El Nitrógeno de la Biomasa Microbiana (NBM) fue calculado por la siguiente ecuación:

$$
\mathrm{N}_{\text {microbiano }}\left(\mathrm{mg} \cdot \mathrm{Kg}^{-1}\right)=\frac{\left(\mathrm{N}_{1}-\mathrm{N}_{\tilde{N} \mathrm{I}}\right)}{\mathrm{Kn}}(4)
$$

$\mathrm{NI}=$ nitrógeno de la muestra irradiada; NÑI $=$ nitrógeno de la muestra no irradiada; $\mathrm{Kn}=$ 0.54, para el método de irradiación-extracción del NBM.

Procesamiento de datos y análisis: Las relaciones entre las emisiones de $\mathrm{CO}_{2} \mathrm{y}$ 
los estratos de vegetación se determinaron con un análisis Kruskal-Wallis y la prueba Tukey, $(\mathrm{P}<0.05)$ y los intervalos de tiempo de incubación se analizaron a través de regresiones representadas en gráficos Kendel Density en el programa PAST3. La estacionalidad y espacialidad de las emisiones y otras variables se analizó a través de análisis de componentes principales (ACP) generando coeficientes de expansión temporal (autovalores) y espacial (autovectores) como patrones de correlación (Ter-Braak, 1986). Se seleccionaron los ACP con sumatoria de $80 \%$ de la varianza total con un $95 \%$ de significancia.

\section{RESULTADOS}

Estacionalidad y espacialidad de las emisiones de $\mathrm{CO}_{2}$ : En la figura 1 se muestra la estacionalidad de las emisiones de $\mathrm{CO}_{2}$ durante 2016/2017 en las 9 parcelas estudiadas. Se evidenció que tanto en el periodo lluvioso y menos lluvioso, el mangle adulto $(7,8,9)$ liberó la mayor cantidad de $\mathrm{CO}_{2}$, situación contraria ocurrió en las parcelas del mangle joven $(1,2,3)$ donde acontecieron las emisiones menores. Sin embargo, el periodo lluvioso 2017 presentó las mayores emisiones.
En la figura 2 se muestran las emisiones estacionarias del $\mathrm{CO}_{2}$ en los tres períodos estudiados. El período lluvioso 2017 (Fig. 2B) sobresale como la época con las mayores emisiones $\left(0.6-16.1 \mathrm{mg} / 100 \mathrm{~cm}^{3}\right)$. Las correlaciones revelan que las emisiones más importantes acontecieron durante los días 3,10,16 (mangle joven), 3,5,10,14,16 (mangle intermediario) y $3,5,7,10,14$ (mangle adulto) respectivamente. En los períodos menos lluviosos (Fig. 2A, Fig. 2C) sobresale el 2017 con mayores emisiones que el 2016.

Variables ambientales en el manglar: La precipitación presentó mayor acumulado en la estación lluviosa 2017 (Fig. 3A) lo que se corroboró con las mayores anomalías positivas de la precipitación de enero hasta abril (Fig. 3B), esta característica estuvo en concordancia con la mayor altura de la marea (Fig. 3C), favoreciendo el incremento de la actividad microbiana en el suelo particularmente en el estrato adulto localizado cerca del canal de la marea.

Emisiones de $\mathrm{CO}_{2}$ y los estratos de vegetación: La información presentada en la tabla 1 indica que las mayores emisiones de $\mathrm{CO}_{2}$ correspondieron al estrato adulto con

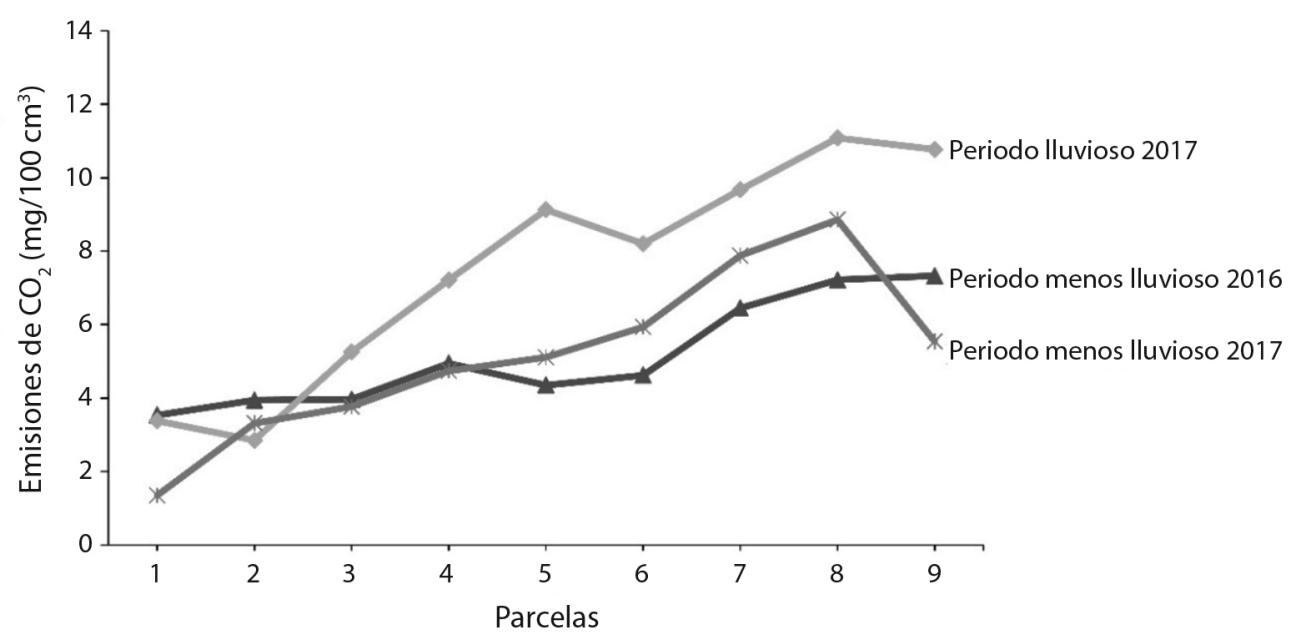

Fig. 1. Emisiones de $\mathrm{CO}_{2}$ en 9 parcelas en los periodos menos lluviosos 2016/2017 (P.M. Lluvioso) y el periodo lluvioso 2017 (P. Lluvioso).

Fig. 1. $\mathrm{CO}_{2}$ emissions in 9 plots in the less rainy periods 2016/2017 (P.M. Rainy) and the rainy season 2017 (P. Rainy). 

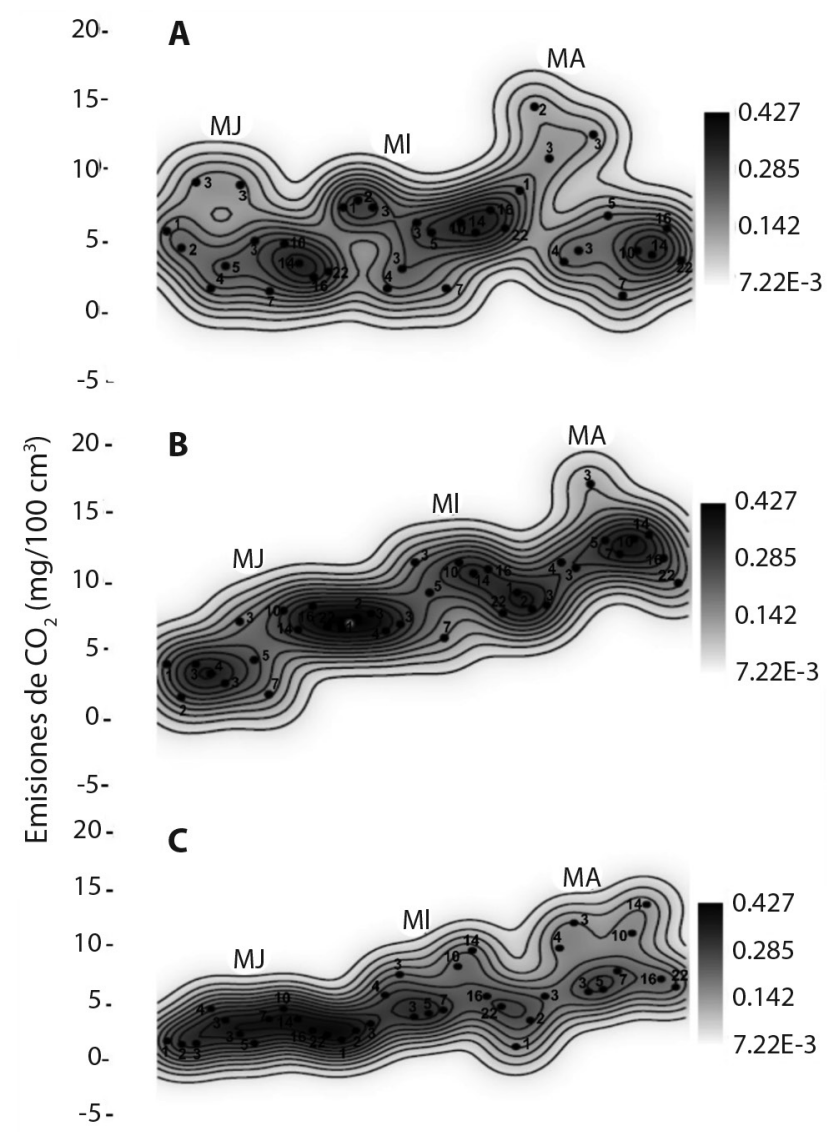

Fig. 2. Correlaciones espaciales y temporales de las emisiones estacionales del $\mathrm{CO}_{2}$. (A) Periodo menos 1luvioso 2016, (B) Periodo lluvioso 2017, (C) Periodo menos lluvioso 2017. Puntos negros representan intervalos de incubación, líneas negras son isolinas de correlación.

Fig. 2. Spatial and temporal correlations of seasonal $\mathrm{CO}_{2}$ emissions. (A) Less rainy period 2016, (B) rainy season 2017, (C) less rainy period 2017 (C). Black dots represent incubation intervals, black lines are correlation isolines.

TABLA 1

Emisiones medias estacionales de dióxido de carbono en tres estratos de mangle con desviación estándar y N poblacional

TABLE 1

Seasonal average emissions of carbon dioxide in three mangrove strata with Standard deviation and population $\mathrm{N}$

\begin{tabular}{lcccc} 
& $\mathrm{N}$ & $\begin{array}{c}\text { Mangle } \\
\text { Joven }\left(\mathrm{TCO}_{2} \text { ha/año }\right)\end{array}$ & $\begin{array}{c}\text { Mangle } \\
\text { Intermediario ( } \mathrm{TCO}_{2} \text { ha/año) }\end{array}$ & $\begin{array}{c}\text { Mangle } \\
\text { Adulto ( } \mathrm{TCO}_{2} \text { ha/año) }\end{array}$ \\
$\begin{array}{lccc}\text { Períodos } \\
\text { Menos Lluvioso 2016 }\end{array}$ & 18 & $5.3 \pm 2.5$ & $9.8 \pm 1.7$ & $13.9 \pm 3.4$ \\
Lluvioso 2017 & 18 & $7.6 \pm 0.5$ & $15.0 \pm 0.5$ & $21.0 \pm 0.9$ \\
Menos Lluvioso 2017 & 18 & $7.6 \pm 3.1$ & $9.9 \pm 2.9$ & $15.0 \pm 1.5$ \\
Promedio & & 6.8 & 11.6 & 16.7 \\
\hline
\end{tabular}

Promedio \pm Desviación estándar. 

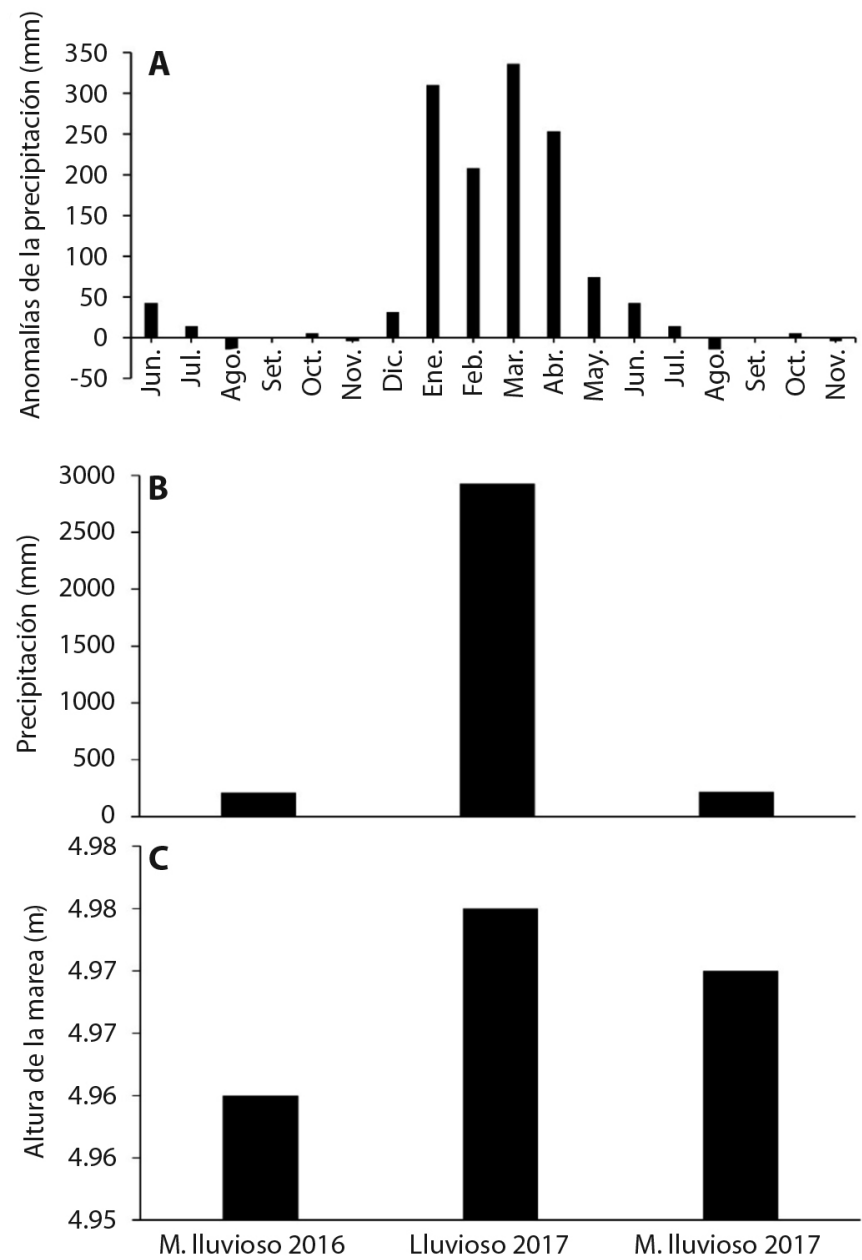

Fig. 3. Variables ambientales. (A) Anomalías mensuales de la precipitación, (B) Acumulado de las precipitaciones, (C) Altura de la marea, período menos lluvioso y lluvioso 2016/2017.

Fig. 3. Environmental variables. (A) Monthly anomalies of precipitation, (B) Accumulated precipitation, (C) Height of the tide, less rainy period and rainy 2016/2017.

16.7 $\mathrm{TCO}_{2}$ ha/año, contrario al estrato joven que liberó $6.8 \mathrm{TCO}_{2}$ ha/año. La prueba de Kruskal-Wallis indicó diferencia significativa entre las emisiones de $\mathrm{CO}_{2}$ y los estratos de vegetación $(\mathrm{P}=0.0170)$, y el test de Tukey confirmó importancia estadística de las emisiones del mangle adulto en relación con los otros estratos $(\mathrm{P}=0.0140)$ en el periodo lluvioso. En el período lluvioso se liberó la mayor cantidad de $\mathrm{CO}_{2}\left(21.0 \pm 0.95 \mathrm{TCO}_{2}\right.$ ha/año $)$ en comparación con los períodos menos lluviosos 2016/2017 donde la humedad del suelo se convirtió en la variable determinante (Fig. 4). Las menores emisiones se registraron en el estrato joven que se caracteriza por estar cerca de la tierra firme y poco cubierta por la marea.

\section{Gradientes estacionales de las emisio-} nes de $\mathrm{CO}_{2}$ y variables físico-químicas: Las correlaciones de emisiones de $\mathrm{CO}_{2}$ y las variables físico-químicas indican que el $56 \%$ de la muestra total está representada en la CP1 y el $24 \%$ en la CP2 (Fig. 4). En la CP1 (Fig. 4) se observa un gradiente diagonal 

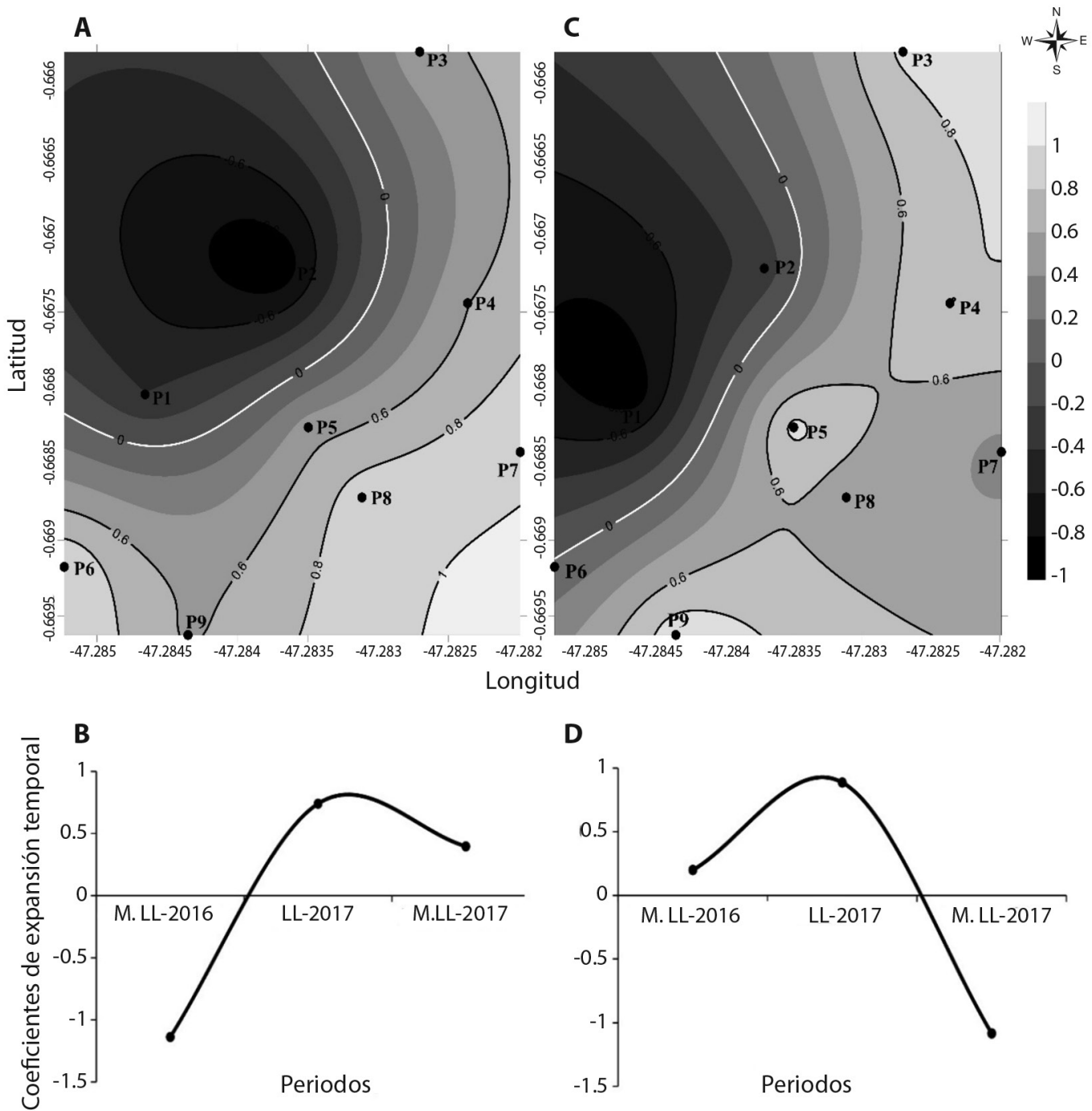

Fig. 4. Representación espacial y temporal de las emisiones de $\mathrm{CO}_{2}$ y variables físico-químicas. (A) Componente principal 1 con $56 \%$ de la varianza total, (B) Coeficiente de expansión temporal en el período menos lluviosos (M. LL), (C) Componente principal 2 con $24 \%$ de la varianza total, (D) Coeficiente de expansión temporal en el período más lluviosos (LL).

Fig. 4. Spatial and temporal representation of $\mathrm{CO} 2$ emissions and physicochemical variables. (A) Principal component 1 with $56 \%$ of the total variance, (B) Coefficient of temporal expansion in the less rainy period (M. LL), (C) Principal component 2 with $24 \%$ of the total variance, (D) Coefficient of temporal expansion in the rainy period (LL).

de sudeste-noroeste, con altas correlaciones, representando las superficies del mangle adulto al sureste y mangle joven al noroeste. En el período lluvioso las parcelas 6,7 y 8 presentaron las mayores emisiones en el mangle adulto; situación inversa ocurre en el período menos lluvioso de 2016, con mayores emisiones de
$\mathrm{CO}_{2}$ en el estrato que en el adulto. En el $\mathrm{CP} 2$ (Fig. 4C, Fig. 4D), se observa un patrón con altas correlaciones sur-noroeste. En el período lluvioso, las parcelas 4,5 y 9 presentaron las mayores emisiones en el mangle intermediario y adulto. Entre las variables más correlacionadas están: la humedad en el suelo (0.89), pH 
$(\mathrm{P}=0.83)$, materia orgánica $(\mathrm{P}=0.8), \mathrm{CBM}(\mathrm{P}$ $=0.87)$ y NBM $(\mathrm{P}=0.89)$.

\section{DISCUSIÓN}

La precipitación acumulada en el 2017 (2 $816 \mathrm{~mm}$ ) sobresalió con relación al 2016 (1 670 $\mathrm{mm}$ ). Al descartarse la estación lluviosa 2017 (2 $597 \mathrm{~mm}$ ) y anomalías positivas durante 4 meses favorecieron el incremento de la actividad microbiana en el suelo y por consiguiente las emisiones de $\mathrm{CO}_{2}$. Las mayores correlaciones estacionales de las emisiones relacionadas con los intervalos de incubación ocurrieron en el estrato adulto los días 3,5,7,10,14, favorecido por la mayor disponibilidad de humedad tanto por los acumulados de las precipitaciones y como por la altura de la marea $(4.9 \mathrm{~m})$.

Este resultado concuerda con un estudio similar de emisiones de $\mathrm{CO}_{2}$ en un bosque secundario de mangle, dominado por el género Avicennia, en el este de Tailandia donde encontraron mayores valores medios de emisiones en el período lluvioso (Poungparn et al., 2009). De igual manera en un estudio realizado por (Valentini, Abreu, \& Faria, 2015), al medir las emisiones de $\mathrm{CO}_{2}$ en el Parque Estadual Massairo Okamura (Mato Grosso) corroboró la correlación de la respiración del suelo con la precipitación y la humedad en el suelo. Ya que la humedad favorece las reacciones bioquímicas de los microorganismos en la época lluviosa, acrecienta las emisiones de $\mathrm{CO}_{2}$. Sin embargo, Mukherjee y Ray (2012) estudiando variaciones mensuales de emisiones de $\mathrm{CO}_{2}$ en manglares de Sundarban, India encontraron que los suelos de manglar liberan más carbono en los meses del período de transición al período lluvioso.

Espacialmente se encontraron mayores emisiones medias en el estrato adulto con promedio de $16.7 \mathrm{TCO}_{2}$ ha/año, contrario al estrato joven que liberó $6.8 \mathrm{TCO}_{2}$ ha/año. En una investigación de manglares mexicanos encontraron valores medios de emisiones de $\mathrm{CO}_{2}$ hasta 6.7 $\mathrm{TCO}_{2}$ ha/año (Silveira et al., 2016). Resultados similares fueron reportados por Nóbrega et al. (2016) en el estudio de emisiones de $\mathrm{CO}_{2}$ en manglares en Ceará, Brasil donde registraron hasta $4 \mathrm{TCO}_{2}$ ha/año. Sin embargo, en los manglares de Rhizophora y Avicennia en la costa venezolana, encontraron tasas de emisión de $11.2 \mathrm{TCO}_{2}$ ha/año (Otero et al., 2017). Otros estudios de las emisiones de $\mathrm{CO}_{2}$ en manglares en la bahía de Gazi, Kenia por Lang'at et al. (2014), dominados por Rhizophora sp., encontraron emisiones de $25.4 \mathrm{TCO}_{2}$ ha/año. Das, Ganguly, Ray, Jana, y Tarun Kumar (2017) por su parte reportaron bajas emisiones de $\mathrm{CO}_{2}$ en manglares tropicales de Sundarban, India, con valores medios de entre 0.23 a $1.94 \mathrm{TCO}_{2}$ ha/año. Así mismo, Poungparn et al. (2009), en el este de Tailandia encontraron valores medios de 3.7 y $4.3 \mathrm{TCO}_{2}$ ha/año respectivamente.

De las variables físico-químicas se destacan la humedad en el suelo, CBM, M.O, pH, salinidad y contenidos de limo y arcilla correlacionados con las emisiones principalmente en el estrato adulto próximo al canal de la marea. Argumentos de Reef et al. (2010) plantean que las propiedades físico-químicas de los suelos del manglar están asociadas a la frecuencia de inundación por la marea. Esta argumentación es concordante con Souza et al. (1996) que determinaron que los niveles de nutrientes en los bosques de manglares generalmente varían de acuerdo con las mareas y el grado de saturación del agua sedimentaria, lo que puede afectar la disponibilidad de los atributos del suelo. Martins y Matthes (1978) señalan que las características del suelo son claves en el comportamiento de los indicadores de calidad del suelo señalando que suelos arenosos, predominantes en mangle joven, son pobres en humedad y materia orgánica que puede significar menores emisiones de $\mathrm{CO}_{2}$.

Con el $56 \%$ de la varianza total de los datos en la ACP1 se observa un gradiente diagonal de sudeste-noroeste que muestra las superficies del mangle adulto al sureste del área de estudio y mayores cantidades de carbono. Mientras que el mangle joven está ubicado al noroeste con bajos índices de humedad y materia orgánica. Las emisiones de dióxido de carbono de los manglares dependen del tamaño del depósito 
de carbono orgánico en el suelo y de las tasas de remineralización (Lovelock et al., 2017).

Los estudios de Lallier-Vergès, Marchand, Disnar, y Lottier (2008), sugieren que el factor marea puede influir en las emisiones de $\mathrm{CO}_{2}$ al alterar el estado de oxidación y los procesos de descomposición de la metería orgánica. De igual manera Keuskamp, Hefting, Dingemans, Verhoeven y Feller (2015) concluyeron que la descomposición microbiana depende de las condiciones ambientales del suelo. En el caso de los manglares se desempeña en gran parte por el régimen de mareas predominante. Los factores como la cantidad de materia orgánica, presencia de agua/humedad y la temperatura influyen en las emisiones de $\mathrm{CO}_{2}$, características que fueron propicias en el período lluvioso (Liblik, Moore, Bubier, \& Robinson, 1997).

La distribución espacial y temporal de las emisiones de $\mathrm{CO}_{2}$ sugiere que la relación emisiones-estratos de vegetación está influenciada por características físicas, químicas y las condiciones ambientales en cada estrato de vegetación principalmente en el periodo lluvioso donde las emisiones de $\mathrm{CO}_{2}$ son en promedio de $14.5 \mathrm{TCO}_{2}$ ha/año. El estrato adulto favoreció el incremento de las emisiones estacionales de $\mathrm{CO}_{2}$ por tener mayor contenido de humedad, tanto por las precipitaciones acumulados de $2595 \mathrm{~mm}$ y altura de la marea de 4.9 $\mathrm{m}$. Principalmente en el período lluvioso esto aumentó la actividad microbiana favoreciendo el incremento en los contenidos de las variables químicas en el suelo y por consiguiente generando mayores emisiones.

Declaración de ética: los autores declaran que todos están de acuerdo con esta publicación y que han hecho aportes que justifican su autoría; que no hay conflicto de interés de ningún tipo; y que han cumplido con todos los requisitos y procedimientos éticos y legales pertinentes. Todas las fuentes de financiamiento se detallan plena y claramente en la sección de agradecimientos. El respectivo documento legal firmado se encuentra en los archivos de la revista.

\section{AGRADECIMIENTOS}

Los autores agradecen a, Coordenação de Aperfeiçoamento de Pessoal de Nível Superior (CAPES) por el apoyo financiero para el primer autor y al Museo Paraense Emilio Goeldi (MPEG) por la asistencia en los análisis de laboratorio.

\section{RESUMEN}

Introducción: Las emisiones de dióxido de carbono de los suelos de manglar tienen un impacto potencial en el balance global de carbono. Objetivo: Investigar la estacionalidad de las emisiones de dióxido de carbono relacionadas con diferentes estratos de la vegetación de manglar, así como las variables físico-químicas del suelo y las ambientales. Métodos: Se demarcaron nueve parcelas de 20 x 20 m (tres para cada estrato de vegetación). Las emisiones de dióxido de carbono fueron monitoreadas durante 88 días a través de la metodología de respiración basal del suelo. Resultados: Los resultados mostraron que las mayores emisiones de dióxido de carbono se presentaron en la temporada lluviosa 2017 con $21.8(7.3 \pm 3.3) \mathrm{mg} / 100 \mathrm{~cm}^{3}$ y $15.7(5.2 \pm 1.6) \mathrm{mg} / 100 \mathrm{~cm}^{3}$ en el período menos lluvioso. En análisis de componentes principales con un $56 \%$ de varianza total, demostró que las mayores correlaciones de las emisiones de dióxido de carbono y las variables físicoquímicas se dieron en el estrato adulto; la humedad del suelo, $\mathrm{pH}$, materia orgánica, carbono y nitrógeno microbiano fueron las variables más correlacionadas. La prueba Kruskal-Wallis corroboró estos resultados comprobando diferencias significativas entre los estratos de vegetación y las emisiones de $\mathrm{CO}_{2}(\mathrm{P}=0.0170)$, y la prueba de Tukey confirmó mayor importancia estadística del manglar adulto en relación con los otros estratos $(\mathrm{P}=0.0140)$. Conclusión: En las tres estaciones analizadas, las mayores emisiones acontecieron en el periodo lluvioso con promedio de 14.5 $\mathrm{TCO}_{2}$ ha/año y el estrato adulto fue el responsable de las mayores emisiones, registrando $21 \mathrm{TCO}_{2}$ ha/año diferencia que fue estadísticamente significativa con los otros estratos $(\mathrm{P}=0.0140)$.

Palabras clave: cambio climático; ecosistema; flujo de carbono; microorganismos sedimentos.

\section{REFERENCIAS}

Alongi, D. (2012). Carbon sequestration in mangrove forests. Carbon Management, 3(3), 313-322. DOI: https://doi.org/doi: $10.4155 / \mathrm{cmt} .12 .20$

Anderson, T. H., \& Domsch, K. H. (1993). The metabolic quotient for $\mathrm{CO} 2(\mathrm{qCO} 2)$ as a specific activity parameter to assess the effects of environmental conditions, 
such as ph, on the microbial biomass of forest soils. Soil Biology and Biochemistry, 25(3), 393-395. DOI: https://doi.org/10.1016/0038-0717(93)90140-7

Berrêdo, J. F., Costa, M. L. da, Vilhena, M. do P. S. P., \& Santos, J. T. dos. (2018). Mineralogia e geoquímica de sedimentos de manguezais da costa amazônica: o exemplo do estuário do rio Marapanim (Pará). Revista Brasileira de Geociências, 38(1), 24-35. DOI: https://doi.org/10.25249/0375-7536.20083812435

Bond-Lamberty, B., \& Thomson, A. (2010). A global database of soil respiration data. Biogeosciences, 7(6), 1915-1926. DOI: https://doi.org/10.5194/ bg-7-1915-2010

Das, S., Ganguly, Ray, R., Jana, T. K., \& Tarun Kumar, D. (2017). Microbial activity determining soil CO emission in the Sundarban mangrove forest, India. Tropical Ecology, 58(3), 525-537.

De Souza Costa, F., Bayer, C., Zanatta, J. A., \& Mielniczuk, J. (2008). Estoque de carbono orgânico no solo e emissões de dióxido de carbono influenciadas por sistemas de manejo no sul do Brasil. Revista Brasileira de Ciencia Do Solo, 32(1), 323-332.

Donato, D. C., Kauffman, J. B., Murdiyarso, D., Kurnianto, S., Stidham, M., \& Kanninen, M. (2011). Mangroves among the most carbon-rich forests in the tropics. Nature Geoscience, 4(5), 293-297. DOI: https://doi. org/10.1038/ngeo1123

Doran, J. W., \& Parkin, T. B. (1994). Defining an assesing soil quality. In J. W. Doran, \& T. B. Parking (Eds.), Defining Soil Quality for a Sustainable Environment (pp. 3-21). Madison, WI, U.S.A.: Soil Science Society of America and American Society of Agronomy.

Fiedler, S. R., Buczko, U., Jurasinski, G., \& Glatzel, S. (2015). Soil respiration after tillage under different fertiliser treatments - implications for modelling and balancing. Soil and Tillage Research, 150, 30-42. DOI: https://doi.org/10.1016/j.still.2014.12.015

Holguin, G., Vazquez, P., \& Bashan, Y. (2001). The role of sediment microorganisms in the productivity, conservation, and rehabilitation of mangrove ecosystems: An overview. Biology and Fertility of Soils, 33(4), 265-278. DOI: https://doi.org/10.1007/ s003740000319

Houghton, R. A. (2003). Why are differences in the terrestrial carbon balance so different? Global Change Biology, 9(4), 500-509.

Joyce, R. J., Janowiak, J. E., Arkin, P. A., \& Xie, P. (2004). CMORPH: A Method that Produces Global Precipitation Estimates from Passive Microwave and Infrared Data at High Spatial and Temporal Resolution. Journal of Hydrometeorology, 5, 487-503.

Keuskamp, J. A., Hefting, M. M., Dingemans, B. J. J., Verhoeven, J. T. A., \& Feller, I. C. (2015). Effects of nutrient enrichment on mangrove leaf litter decomposition. Science of the Total Environment, 508, 402-410.

Kim, D. G., Vargas, R., Bond-Lamberty, B., \& Turetsky, M. R. (2012). Effects of soil rewetting and thawing on soil gas fluxes: A review of current literature and suggestions for future research. Biogeosciences, 9(7), 2459-2483. DOI: https://doi.org/10.5194/ bg-9-2459-2012

Lallier-Vergès, E., Marchand, C., Disnar, J. R., \& Lottier, N. (2008). Origin and diagenesis of lignin and carbohydrates in mangrove sediments of Guadeloupe (French West Indies): Evidence for a two-step evolution of organic deposits. Chemical Geology, 255, 388-398. DOI: https://doi.org/10.1016/j.chemgeo.2008.07.009

Lang'at, J. K. S., Kairo, J. G., Mencuccini, M., Bouillon, S., Skov, M. W., Waldron, S., \& Huxham, M. (2014). Rapid losses of surface elevation following tree girdling and cutting in tropical mangroves. PLoS ONE, 9, 1-8. DOI: https://doi.org/10.1371/journal. pone. 0107868

Liblik, L. K., Moore, T. ., Bubier, J. L., \& Robinson, S. D. (1997). Methane emissions from wetlands in the zone of discontinuous permafrost: Fort Simpson, Northwest Territories, Canada. Global Biogeochemical Cycles, 11(4), 485-494.

Lovelock, C. E., Atwood, T., Baldock, J., Duarte, C. M., Hickey, S., Lavery, P. S., ... Steven, A. (2017). Assessing the risk of carbon dioxide emissions from blue carbon ecosystems. Frontiers in Ecology and the Environment, 15(5), 257-265. DOI: https://doi. org/10.1002/fee. 1491

Martins, F. R., \& Matthes, L. A. F. (1978). Respiração edáfica e nutrientes na Amazônia (Região de Manaus): floresta arenícola, campinarana e campina. Acta Amazonica, 8(2), 233-244.

de Sá Mendonça, E., \& da Silva Matos, E. (2005). Matéria orgânica do solo: métodos de análises. Brasil: ABEAS.

Mukherjee, J., \& Ray, S. (2012). Carbon cycling from mangrove litter to the adjacent Hooghly estuary, India - A modelling study. Procedia Environmental Sciences, 13, 391-413. DOI: https://doi.org/10.1016/j. proenv.2012.01.036

Nóbrega, G. N., Ferreira, O. T., Siqueira Neto, M., Queiroz, H. M., Artur, A. G., \& Otero, X. L. (2016). Edaphic factors controlling summer (rainy season) greenhouse gas emissions (CO2 and $\mathrm{CH} 4)$ from semiarid mangrove soils (NE-Brazil). Science of the Total Environment, 542, 685-693. DOI: https://doi.org/10.1016/j. scitotenv.2015.10.108

Otero, X. L., Méndez, A., Nóbrega, G. N., Ferreira, T. O., Santiso-Taboada, M. J., Meléndez, W., \& Macías, F. (2017). High fragility of the soil organic C 
pools in mangrove forests. Marine Pollution Bulletin, 119(1), 460-464. https://doi.org/10.1016/j. marpolbul.2017.03.074

Poungparn, S., Komiyama, A., Tanaka, A., Sangtiean, T., Maknual, C., Kato, S., ... Patanaponpaiboon, P. (2009). Carbon dioxide emission through soil respiration in a secondary mangrove forest of eastern Thailand. Journal of Tropical Ecology, 25(4), 393-400. DOI: https://doi.org/10.1017/s0266467409006154

Reef, R., Feller, I. C., \& Lovelock, C. E. (2010). Nutrition of mangroves. Tree Physiology, 30(9), 1148-1160. DOI: https://doi.org/10.1093/treephys/tpq048

Schaeffer-Novelli, Y., Cintrón-Molero, G., Adaime, R. R., de Camargo, T. M., Cintron-Molero, G., \& de Camargo, T. M. (1990). Variability of Mangrove Ecosystems along the Brazilian Coast. Estuaries, 13(2), 204-218.DOI: https://doi.org/10.2307/1351590

Sifleet, S., Pendleton, L., \& Murray, B. (2011). State of the Science on Coastal Blue Carbon A Summary for Policy Makers (Technical report). U.S.A.: Nicholas Institute for Environmental Policy Solutions-Duke University.

Silveira, J. A. H., Rico, A. C., Pech, E., Pech, M., Ramírez, J., \& Teutli, C. (2016). Carbon Dynamics ( Stocks and Fluxes ) in Mangroves of Mexico. Terra Latinoamericana, 34(1), 61-72.
Souza, H. F., Guedes, M. L. S., Oliveira, S. S. de, \& Santos, E. S. (1996). Alguns aspectos fitossociológicos e nutricionais do manguezal da Ilha de Patí-Bahía, Brasil. Sitientibus, Feira de Santana, 15, 151-165.

Stockmann, U., Adams, M. A., Crawford, J. W., Field, D. J., Henakaarchchi, N., Jenkins, M., ... Zimmermann, M. (2013). The knowns, known unknowns and unknowns of sequestration of soil organic carbon. Agriculture, Ecosystems and Environment, 164, 80-99. DOI: https://doi.org/10.1016/j.agee.2012.10.001

Ter-Braak, C. J. (1986). Canonical correspondence analysis: a new technique for multivariate direct gradient analysis. Ecological Society of America, 67(5), 1167-1179.

Troxler, T. G., Barr, J. G., Fuentes, J. D., Engel, V., Anderson, G., Sanchez, C., \& Davis, S. E. (2015). Component-specific dynamics of riverine mangrove $\mathrm{CO} 2$ efflux in the Florida coastal Everglades. Agricultural and Forest Meteorology, 213, 273-282. DOI: https:// doi.org/10.1016/j.agrformet.2014.12.012

Valentini, C. M. A., Abreu, J. G. de, \& de Faria, R. A. P. G. (2015). Respiração Do Solo Como Bioindicador Em Áreas Degradadas. Revista Internacional de Ciências, 5(2), 127-143. DOI: https://doi.org/10.12957/ ric. 2015.19581 